\title{
Downstream Targets of Homeobox Gene HLX Show Altered Expression in Human Idiopathic Fetal Growth Restriction
}

\author{
Gayathri Rajaraman, Padma Murthi, \\ Niroshani Pathirage, Shaun P. Brennecke, \\ and Bill Kalionis

\begin{abstract}
From the Department of Obstetrics and Gynaecology, University of Melbourne and Pregnancy Research Centre, The Royal
\end{abstract} \\ Women's Hospital, Parkville, Australia
}

Fetal growth restriction (FGR), a clinically significant pregnancy disorder, is poorly understood at the molecular level. This study investigates idiopathic FGR associated with placental insufficiency. Previously, we showed that the homeobox gene $H L X$ is expressed in placental trophoblast cells and that $H L X$ expression is significantly decreased in human idiopathic FGR. Here, we used the novel approach of identifying downstream targets of $H L X$ in cell culture to detect potentially important genes involved in idiopathic FGR. Downstream targets were revealed by decreasing $H L X$ expression in cultured trophoblast cells with $H L X$-specific small interfering RNAs to model human idiopathic FGR and comparing these levels with controls using a real-time PCR-based gene profiling system. Changes in candidate $H L X$ target mRNA levels were verified in an independent trophoblast cell line, and candidate target gene expression was assessed in human idiopathic FGR-affected placentae $(n=25)$ compared with gestation-matched controls $(n=25)$. The downstream targets $R B 1$ and $M Y C$, cell cycle regulatory genes, showed significantly increased mRNA levels in FGR-affected tissues compared with gestation-matched controls, whereas CCNB1, ELK1, $J U N$, and $C D K N 1$ showed significantly decreased mRNA levels $(n=25, P<0.001, t$-test $)$. The changes for $R B 1$ and $C D K N 1 C$ were verified by Western blot analysis in FGR-affected placentae compared with gestation-matched controls $(n=6)$. We conclude that cell cycle regulatory genes $R B 1, M Y C, C C N B 1$, ELK1,$J U N$, and CDKN1C, which control important trophoblast cell functions, are targets of $H L X$. (Am J Pathol 2010, 176:278-287; DOI: 10.2353/ajpath.2010.090187)
Fetal growth restriction (FGR), also known as intrauterine growth retardation, is a significant pregnancy disorder associated with major perinatal complications including stillbirth, prematurity, fetal compromise, infant morbidity and mortality. Moreover, later in life, FGR-affected individuals also have an increased risk of chronic disorders such as ischemic heart disease, maturity onset diabetes, and psychiatric disorders. ${ }^{1,2}$ A common definition of FGR is a birth weight at or below the 10th percentile for gestational age and gender, failure of the fetus to grow to its genetically determined potential size, and the likely presence of an underlying pathological process that inhibits the expression of the normal intrinsic growth potential. Obvious causes of FGR are fetal (eg, genetic defects), placental (eg, infarcts), and maternal factors (eg, maturity onset diabetes, certain autoantibody disorders, alcohol abuse, and smoking), but these factors account for only $30 \%$ of FGR cases. The remaining $70 \%$ of FGR cases with no obvious causes are classified as "idiopathic" and are commonly attributed to uteroplacental insufficiency.

Placental insufficiency is associated with abnormal trophoblast function in FGR. Villous outgrowth, which is determined by trophoblast proliferation, is reduced in the FGR-affected placenta together with increased apoptosis of these cells. ${ }^{3}$ Defective trophoblast function also results in growth restriction of the fetus due to the reduction in transfer of nutrients and growth factors to the fetus. ${ }^{4}$ Another significant defect in FGR is uteroplacental ischemia due to failure of the specialized extravillous trophoblast cells to proliferate, invade, and subsequently trans-

\footnotetext{
Supported by National Health and Medical Research Council project grant 509140. The authors would also like to thank Lynne Quayle Charitable Trust Fund (Equity Trustees) and the Marian E.H. Flack Trust for funding support for this project and also the University of Melbourne for the award of an Early Career Researcher Grant (to P.M.) G.R was awarded the Felix Meyer Postgraduate Scholarship from the University of Melbourne and the Royal Women's Hospital Postgraduate Scholarship.

Accepted for publication September 18, 2009

Address reprint requests to Gayathri Rajaraman, Ph.D., Department of Obstetrics and Gynaecology, University of Melbourne, RWH Campus, 20 Flemington Rd., Parkville, Victoria 3052, Australia. E-mail: graja@unimelb.edu.au.
} 
form and remodel the maternal spiral arteries in the placental bed. ${ }^{5}$ Villous structural defects, vascular abnormalities, and reduced branching of the villous structure are also seen in FGR. ${ }^{6-8}$ These defects also prevent adequate nutrient transfer to the fetus. ${ }^{9}$

Mouse knockout studies of transcription factors provide genetic proof of the critical role of transcription factors in regulating placental development. ${ }^{10-14}$ Some knockout gene phenotypes show the hallmarks of major human placental disorders ${ }^{13}$ and thus may play an important role in the pathogenesis of human idiopathic FGR.

The homeobox gene family of transcription factors is extensively characterized in the human placenta and embryo. ${ }^{15,16}$ These genes are known to play important roles in implantation, placentation, and embryonic development in the mouse. ${ }^{11-13,17-20}$ Only a few studies of human homeobox genes have provided evidence that homeobox genes are important in human placental development and particularly in trophoblast development and differentiation. ${ }^{10,16,21,22}$ Targeted disruption of the HIx homeobox gene in the mouse has shown that HIx also plays a fundamental role in visceral organogenesis. ${ }^{23} \mathrm{HIX}$ mutant mice resulted in developing gut and liver diverticulum defects. In addition, HIx mutation also showed a defect in proliferation and resulted in embryonic death due to liver failure. ${ }^{23}$

Our interest is in the homeobox gene HLX, the human homologue of mouse HIx homeobox gene, which is highly expressed in hematopoietic progenitors and shows decreased expression levels in activated lymphocytes. ${ }^{24}$ $H L X$ inactivation impairs CD34+ ${ }^{+}$bone marrow cell proliferation in response to stimulation by cytokines while inducing differentiation of these cells. Moreover, $H L X$ inactivation reduces the levels of $c-m y c, c-f o s, c y c l i n B$, and p34 ${ }^{\mathrm{cdc} 2}$ mRNA expression, ${ }^{24}$ which are cell cycle regulator genes implicated in trophoblast cell function. ${ }^{25}$ Therefore, $c-m y c, c-f o s, c y c l i n B$, and $p 34^{c d c 2}$ were the first identified targets of $H L X$.

Slavotinek et $\mathrm{al}^{26}$ have described HLX mutations in human congenital diaphragmatic hernia patients. In this study, the HLX gene was sequenced in 119 congenital diaphragmatic hernia patients, because HLX is in the deleted interval at ch1q41-1q42 for human congenital diaphragmatic hernia, and four amino acid substitution mutations (p.A235V, p.S12F, p.S18L, and p.D173Y) resulted in congenital diaphragmatic hernia phenotype. ${ }^{26}$ Slavotinek et $\mathrm{al}^{26}$ concluded that $H L X$ mutations are etiologically important in human diaphragm formation by interaction with other genetic or environmental factors. Furthermore, Suttner et $\mathrm{al}^{27}$ have determined that $H L X$ gene variants influence the development of childhood asthma. Their study identified 19 polymorphisms in the $H L X$ gene, and two tagging single nucleotide polymorphisms representing seven polymorphisms were associated with childhood asthma in a study population of 3099 German children. Suttner et $\mathrm{al}^{27}$ concluded that genetic alterations in $H L X$ contributed to the pathogenesis of childhood asthma.

Previously, we provided evidence that HLX is expressed primarily in the proliferating cytotrophoblast cell types in early placental development. ${ }^{28}$ Furthermore, we postulated that reduced levels of $H L X$ are required for cytotrophoblast differentiation and that dysregulation of $H L X$ expression contributed to the aberrant cytotrophoblast proliferation and differentiation associated with placental pathologies. ${ }^{28}$ Recently, we also provided evidence of $H L X$ regulation by growth factors and cytokines and established that $H L X$ is an important regulator for signal transduction-mediated proliferation of human trophoblast cells. ${ }^{29}$ By using four independent small interfering RNA (siRNA) oligonucleotides, we have reported a significant 60 to $85 \%$ decrease in HLX mRNA and 73 to $87 \%$ decrease in protein expression by HLX siRNA transfection compared with the mock-transfected control trophoblast cells. ${ }^{29}$ Finally, we showed that HLX mRNA and protein expression is significantly decreased in human idiopathic FGR, ${ }^{30}$ suggesting that $H L X$ expression may be of pathological significance.

The focus of this study is to identify downstream target genes of HLX in cultured trophoblast and to identify the molecular pathways that are affected in human idiopathic FGR.

Signal transduction pathways that regulate trophoblast functions include the phosphatidylinositol 3-kinase-Akt, protein kinase A, protein kinase C, p38/c-Jun N-terminal kinase, Jak-Stat, and mitogen-activated protein kinase (MAPK) pathways. ${ }^{31-33}$ Of these, the two pathways that are frequently used by trophoblast cells are the Jak-Stat and MAPK pathways. ${ }^{33}$ The human MAPK signaling pathway PCR array was used in this study.

We hypothesize that placental HLX downstream target gene expression will be significantly altered in human idiopathic FGR pregnancies compared with uncomplicated pregnancies. In this study, an in vitro cell culture model using HLX siRNA, in trophoblast cells, was used. PCR array comparisons were made between HLX siRNA-treated cells and control cells transfected with a siRNA that does not target human genes. Candidate $H L X$ target gene expression levels were then assessed by real-time PCR, using validated probes, in human idiopathic FGR-affected placentae compared with gestation age-matched control placentae.

\section{Materials and Methods}

\section{Patient Details and Tissue Sampling}

Human placentae from pregnancies complicated by idiopathic FGR $(n=25)$ and gestation-matched control pregnancies ( $n=25$ ) were obtained with informed patient consent and with approval from the Research and Ethics committees of The Royal Women's Hospital. These samples have been characterized and used in our previous gene expression studies. ${ }^{30,34}$ Ultrasound was used for the prospective identification of growth-restricted fetuses. The clinical characteristics of FGR-affected pregnancies and the gestation age-matched controls used in this study are as previously published by Murthi et al. ${ }^{30,34}$ As described, ${ }^{30,34}$ the inclusion criteria for this study were a birth weight less than the 10th percentile for 
gestation age using Australian growth charts ${ }^{35}$ and any two of the following criteria diagnosed on antenatal ultrasound: abnormal umbilical artery Doppler flow velocimetry, oligohydramnios as determined by amniotic fluid index $<7$ on antenatal ultrasound performed before delivery, or asymmetric growth of the fetus as quantified from the head circumference-to-abdominal circumference ratio (>1.2). Placental tissue subjected to maternal smoking, chemical dependency, multiple pregnancies, pre-eclampsia, placental abruption, prolonged rupture of the membranes, fetal congenital anomalies, or suspicion of intrauterine viral infection were excluded from this study. Pre-eclampsia cases with associated FGR were also excluded from this study. The last menstrual period dates were used to calculate the gestation age for both FGR and control patients and further confirmed by early pregnancy ultrasound. Control patients were selected to match FGR cases according to gestation. All control patients presented with either spontaneous labor or required elective delivery by induction of labor/cesarean section. Preterm control patients presented in spontaneous idiopathic preterm labor or underwent elective delivery for conditions not associated with placental dysfunction (eg, elective cesarean section due to maternal breast cancer). Particularly, control patients did not have clinical evidence of FGR, pre-eclampsia, placental abruption, prolonged rupture of the membranes, or ascending infection. All control patients gave birth to normally formed babies with birth weights appropriate for gestation. All control placentae obtained were grossly normal with no observed abnormalities such as infarcts.

Placental tissues were excised from randomly selected areas of central placental cotyledons, after dissecting away any attached decidua. Samples were processed within 10 minutes of delivery of the placenta. Samples of fresh placental tissues were divided into small pieces and thoroughly washed in $0.9 \%$ PBS to minimize blood contamination and then snap frozen and stored at $-80^{\circ} \mathrm{C}$ for RNA analysis.

\section{Cell Culture}

Human trophoblast cell lines are a well established model for examining trophoblast function in vitro. In this study, two well-characterized, human extravillous trophoblast cell lines SGHPL-4 and HTR-8/SVneo were used, because these cell lines retain many features of normal extravillous trophoblast cells, including human placental lactogen, human chorionic gonadotropin, and HLAClass $1 .{ }^{36,37}$ The cell lines used in this study are long term, which senesce after $\sim 30$ passages. For this work, SGHPL-4 cells (passages 5 to 12) were cultured in supplemented Ham's F-10 medium, and the HTR-8/Svneo cells (passages 16 to 19) were cultured in supplemented RPMI 1640 medium, as described previously. ${ }^{29}$

\section{Inactivation of $\mathrm{HLX}$ by SiRNA}

Inactivation of HLX by siRNA was performed as previously described. ${ }^{29}$ Briefly, cultured trophoblast cells were grown in Ham's F-10 nutrient mixture/RPMI 1640 medium supplemented with $10 \%$ fetal bovine serum for 48 hours $\left(2 \times 10^{5}\right.$ cells/well in 6 -well plates $)$ and then transfected with two individual HLX siRNAs [HLX-si1 sequence: r(UGAAUUUCUUGGGUUCGAG)d(TT); HLX-si2 sequence: r(AAACCUUUUCUCCAGG CCU)d(TT)], using RNAiFect transfection reagent (Qiagen, Victoria, Australia). The mock-transfected control wells received all reagents, except the siRNA oligonucleotide. Cells were returned to incubation at $37^{\circ} \mathrm{C}$ in $5 \% \mathrm{CO}_{2}$ for 72 hours. Negative control siRNAs consisted of a pool of enzymegenerated siRNA oligonucleotides of 15 to $19 \mathrm{bp}$ in length, which were not specific for any known human gene (catalog number 0652-13-7000, Superarray Bioscience, Frederick, MD) and showed no sequence similarity to $H L X$.

\section{RNA Extraction}

Total RNA from excised placental tissue was isolated as described previously by Murthi et al. ${ }^{30,34}$ Briefly, $300 \mathrm{mg}$ of placental tissue was homogenized, and total RNA was isolated by acid guanidium isothiocyanate-phenol-chloroform extraction and lithium chloride precipitation according to the method of Chomzynski and Sacchi ${ }^{38}$ and as modified by Puissant and Houdebine. ${ }^{39}$ Total cellular RNA from cultured trophoblast cells was isolated using the QIAShredder and an RNeasy Microkit (Qiagen) according to the manufacturer's instructions. First-strand synthesis for real-time PCR cDNA preparation was performed on $2 \mu \mathrm{g}$ of total RNA from placental tissue and cultured cells, using Superscript III ribonuclease H-reverse transcriptase (Invitrogen, Victoria, Australia). Firststrand synthesis of CDNA for $\mathrm{RT}^{2}$-profiler PCR array was performed using the $\mathrm{RT}^{2}$ First Strand kit (catalog number c-03; SuperArray Bioscience). Briefly, $10 \mu$ l of reverse transcriptase mixture (containing reverse transcriptase buffer, primer, external control mix and reverse transcriptase enzyme mix) was added to $10 \mu$ l of genomic DNA elimination mixture (containing $1 \mu \mathrm{g}$ of RNA, gDNA elimination buffer, and RNase-free water) and incubated at $42^{\circ} \mathrm{C}$ for 15 minutes. RNA degradation and reverse transcriptase inactivation were performed by heating at $95^{\circ} \mathrm{C}$ for 5 minutes.

\section{PCR Array}

The downstream target genes of HLX were determined using the real-time PCR based "RT ${ }^{2}$ profiler PCR array" for gene profiling (SuperArray Bioscience Corporation, catalog no. PHAS-061). The human MAPK signaling pathway PCR array was used in this study. This commercially available PCR array encompassed the gene expression profiles of 84 genes related to the MAPK signaling pathway, which is an important signaling pathway in the regulation of human trophoblast function. ${ }^{33}$ Members of the MAP kinase kinase kinase (MKKK), MAP kinase kinase (MKK), and MAPK families are represented on this array as well as transcription factors and genes whose expression is induced by MAPK signaling. In addition, the array also included genes related to scaffolding and anchoring. Furthermore, the PCR array chosen in this 
study contained several cell cycle regulatory genes, including CCNB1, MYC, CDKN1C, and JUN, which have been previously identified to be the targets of HLX in hematopoietic progenitor cells. ${ }^{24}$

Procedures were performed according to the manufacturer's protocol. Briefly, diluted cDNA prepared using the $\mathrm{RT}^{2}$ First-Strand kit (as described above) was added to a master mix containing the fluorescent SYBR green/ ROX dye. Aliquots from this mix were added to a 96-well plate that contained 84 predispensed gene-specific primer sets. The plates (one for each HLX-siRNA and controls) were then placed in an ABI Prism 7700 Sequence Detector (PerkinElmer-Applied Biosystems, Victoria, Australia) for quantitation of gene expression under the following cycling parameters: $95^{\circ} \mathrm{C}$ for 10 minutes, followed by 40 cycles of denaturation at $95^{\circ} \mathrm{C}$ for 15 seconds and primer extension at $60^{\circ} \mathrm{C}$ for 1 minute. Each plate contained a panel of five housekeeping gene primers for normalization of the PCR array data, as well as estimation of the linear dynamic range of the assay. The housekeeping gene panel consisted of $\beta_{2}$-micrglobulin (B2M), hypoxanthine phosphoribosyltansferase 1 (HPRT1), ribosomal protein $\mathrm{L} 13 \mathrm{a}(R P L 13 A)$, glyceraldehyde-3-phosphate dehydrogenase $(G A P D H)$, and $\beta$-actin $(A C T B)$. Furthermore, for each reaction, both negative control (no reverse transcriptase enzyme added) and template control (no cDNA added) were included as controls. Raw data were acquired and processed with $A B I$ Sequence Detector System software version 1.0 (PerkinElmer-Applied Biosystems) to calculate the threshold cycle $\left(C_{t}\right)$ value. Relative gene expression values (fold change) were subsequently determined according to the manufacturer's instructions. Briefly, the fold change for each gene from the mock control array and the HLX-siRNA-treated array was calculated as $2^{-\Delta \Delta C t}$, normalized to the average $C_{t}$ value of the five housekeeping genes. Fold changes $>1$ were fold increase, and for fold changes $<1$, the negative inverse of the result was reported as a fold decrease. Candidate gene prioritization was based on the highest positive and lowest negative fold change differences.

\section{Real-Time PCR}

Verification of candidate $H L X$ target gene mRNA expression (identified from PCR array) in mock control and HLX siRNA-transfected SGHPL-4 and HTR-8/ Svneo cells was performed on an ABI Prism 7700 (PerkinElmer-Applied Biosystems) using prevalidated TaqMan Gene Expression Assays (RB1 Assay ID Hs00153108_m1, EGR1 Assay ID Hs00152928_m1, MYC Assay ID Hs00153408_m1, JUN Assay ID Hs00277190_s1, Cyclin B1 Assay ID Hs00259126_m1, ELK1 Assay ID Hs00428286_g1, and CDKN1C Assay ID Hs00175938_m1; Applied Biosystems). Gene expression quantitation was performed as the second step in a twostep RT-PCR protocol according to the manufacturer's instructions. A total of $20 \mu \mathrm{l}$ of PCR mix containing TaqMan Universal PCR master mix, TaqMan Gene Expression Assay mix and $3.5 \mathrm{ng}$ of cDNA was used. Amplification was performed for 40 cycles, including denaturation at $95^{\circ} \mathrm{C}$ for 15 seconds and annealing/extension at $60^{\circ} \mathrm{C}$ for $60 \mathrm{sec}-$ onds. Gene expression for the housekeeping gene GAPDH was co-amplified with HLX in the same reaction well. Murthi et al ${ }^{34}$ from our laboratory previously showed that GAPDH is a suitable endogenous reference gene for relative gene expression studies in placental tissues from human idiopathic FGR, and its expression is not altered in FGR placentae compared with control placentae. The GAPDH primers (5'-GCACCACCAACTGCTTAGCA-3' and 5'-GTCTTCTGGGTGGCAGTGATG-3') and TaqMan probe (5'-VIC-TCGTGGAAGGACTCATGACCACAGTCC-TAMRA$\left.3^{\prime}\right)$ were designed using Primer Express 1.5 software (Applied Biosystems). The cycling conditions for GAPDH were as per the $H L X$ target genes. Relative quantitation of $H L X$ target gene expression normalized to GAPDH was calculated according to the $2^{-\Delta \Delta C T}$ method of Livak and Schmittgen ${ }^{40}$ using the mock control as a calibrator (ABI Prism 7700 Sequence detection system, User Bulletin number 2, 2001). These candidate HLX target genes were then further tested for expression in FGR-affected

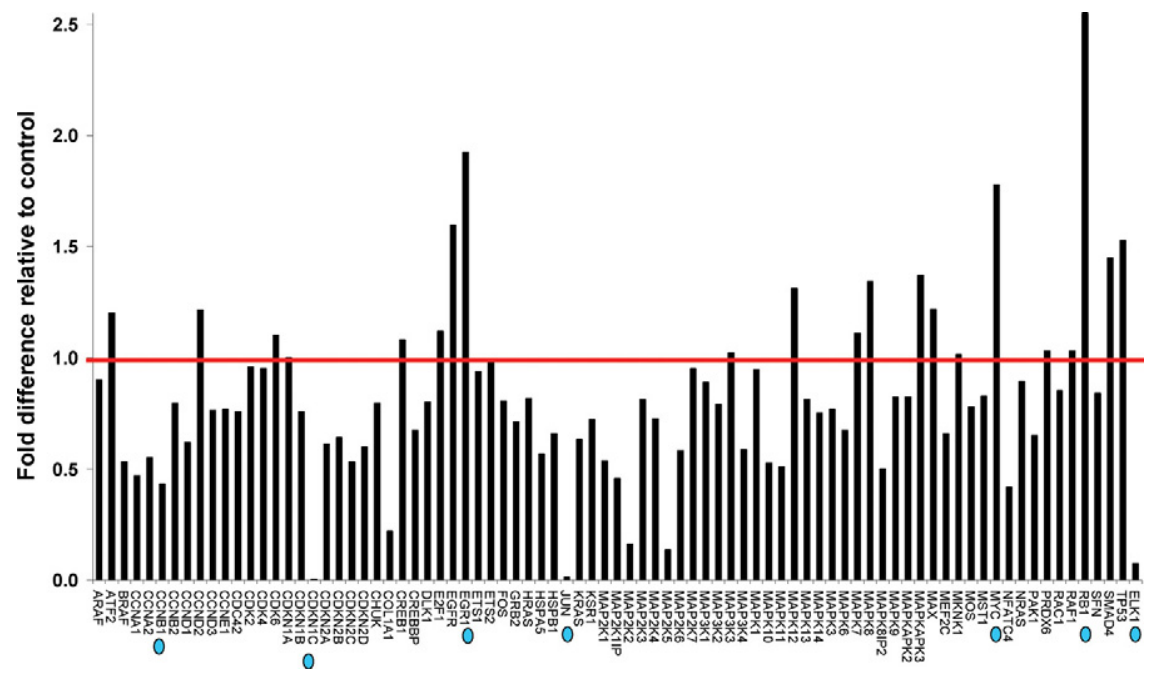

Figure 1. Identification of $H L X$ downstream target genes. RNA was extracted from SGHPL-4 cells transfected with $H L X$ siRNA, transcribed into first strand CDNA, and the $\mathrm{RT}^{2}$ profiler PCR array was performed for gene profiling. Following initial denaturation at $95^{\circ} \mathrm{C}$ for 10 minutes, the 84 predispensed genes and a panel of housekeeping genes were amplified for 40 cycles including denaturation at $95^{\circ} \mathrm{C}$ for 15 seconds and primer extension at $60^{\circ} \mathrm{C}$ for 1 minute. Relative gene expression values (fold change above or below threshold value of 1) were subsequently calculated for the $H L X$ siRNA-treated plate, relative to the mock control plate, normalized to the housekeeping gene panel. The red line shows the threshold value at 1 . The prioritized candidate $H L X$ target genes are indicated by blue dots 
human placental samples compared with gestation agematched controls, as per the protocol described above.

\section{Western Immunoblotting}

Samples were electrophoresed on a 10\% PAGE/0.1\% SDS gel in running buffer $(250 \mathrm{mmol} / \mathrm{L}$ Tris and $1.92 \mathrm{mmol} / \mathrm{L}$ glycine) and transferred electrophoretically to a nitrocellulose membrane at $100 \mathrm{~V}$ for 30 minutes in transfer buffer (255 mmol/L Tris, $1.92 \mathrm{mmol} / \mathrm{L}$ glycine, and 0.1\% SDS). The filter was blocked with $10 \%$ (w/v) nonfat milk powder/TBS for 1 hour. Incubation with the RB1 $(0.5 \mu \mathrm{g} / \mathrm{ml}), C D K N 1 C$ (1 $\mu \mathrm{g} / \mathrm{ml}$ ) (Sapphire Biosciences, New South Wales, Australia) or GAPDH $(0.5 \mu \mathrm{g} / \mathrm{ml})$ (Imgenex, San Diego, CA) antibodies was performed overnight at $4^{\circ} \mathrm{C}$. The filter was then washed in Tris-buffered saline and incubated with biotinylated antirabbit antibody $(1.5 \mu \mathrm{g} / \mathrm{ml}$ ) (Amersham Life Sciences, New South Wales, Australia) in 5\% (w/v) nonfat milk powder/TBS for at least 1 hour at room temperature. Streptavidin-HRP $(1.5 \mu \mathrm{g} / \mathrm{ml})$ in $2 \%$ nonfat milk powder/Tris-buffered saline buffer was added and incubation was performed at room temperature for 1 hour. Tyramide signal amplification was then performed, according to the manufacturer's instructions (PerkinElmer), for CDKN1C blots. RB1 and GAPDH antibodies did not require tyramide signal amplification. Detection of protein bands was performed using ECL-Lumilyte autoradiography kit according to the manufacturer's instructions (PerkinElmer).

\section{Data Analysis}

All parameters for the gestation age-matched, FGR-affected pregnancies and controls were described as mean \pm SEM. Differences between the clinical characteristics of the FGR-affected pregnancies and the control patients were investigated using either $\chi^{2}$ test or Student's $t$-test where appropriate. The difference in mRNA expression of the HLX target genes between siRNA-treated and control-cultured trophoblast cells and between FGR-affected and control pregnancies was assessed by $t$-test. A value of $P<0.001$ was considered significant.

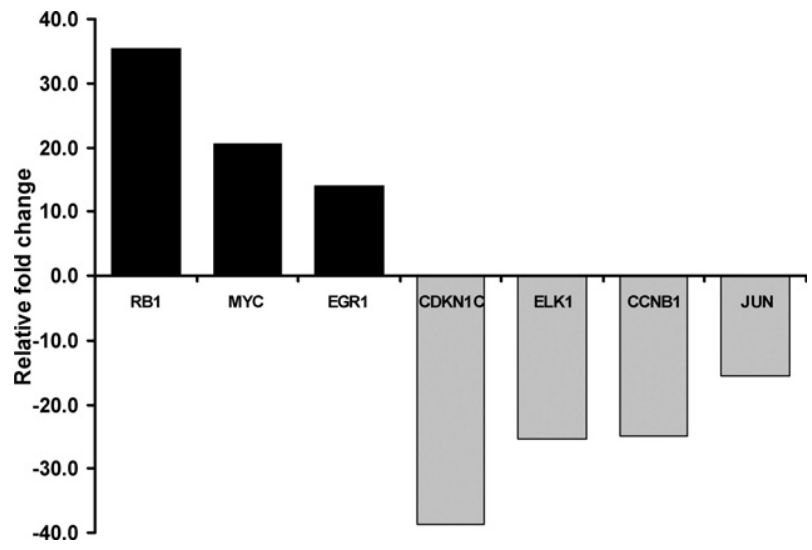

Figure 2. Prioritized $H L X$ downstream target genes. $H L X$ downstream target genes, as identified in Figure 1, were prioritized on the basis of most increased and most decreased gene expression with HLX siRNA transfection, compared with the control. Fold changes greater than 1 were reported as fold increase, and for fold changes $<1$, the negative inverse of the result was reported as a fold decrease.

\section{Results}

\section{Identification of HLX Downstream Targets}

The SGHPL-4 human trophoblast cell line was transfected with two independent siRNA oligonucleotides de-

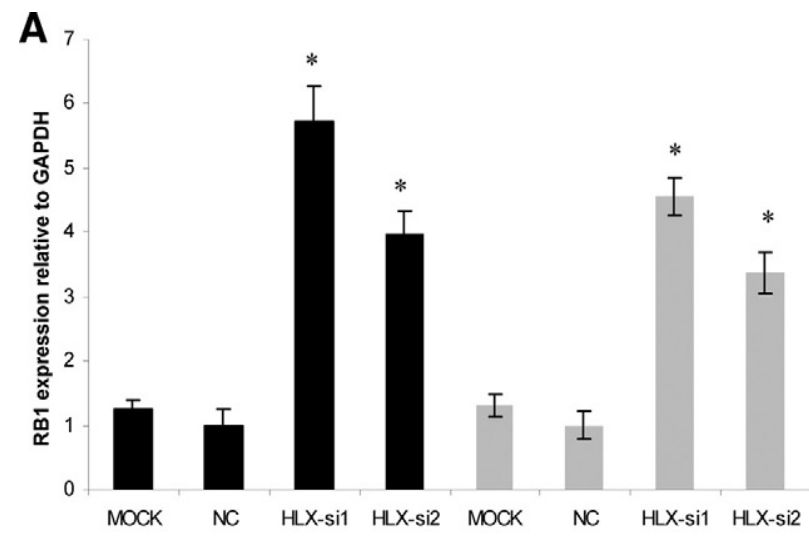

B

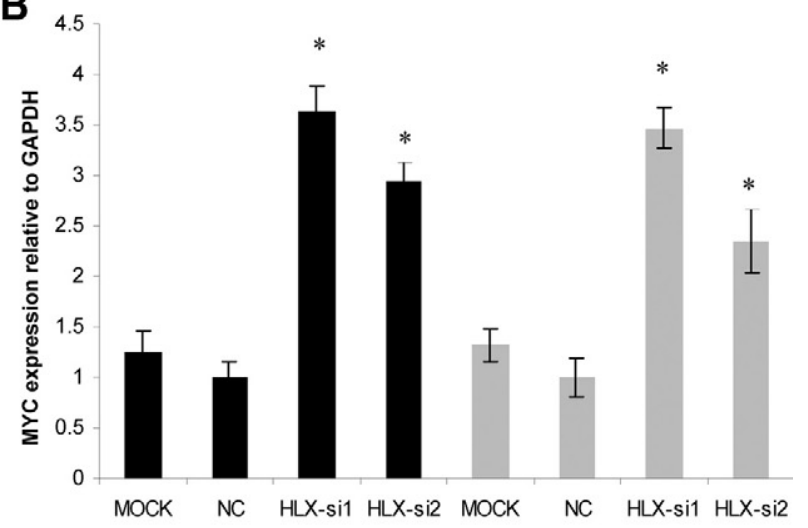

C

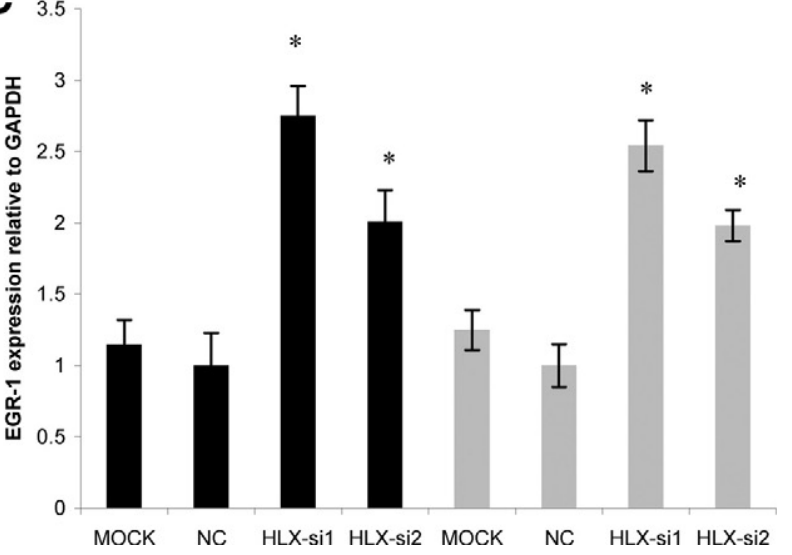

Figure 3. Validation of target genes that showed increased expression following siRNA-mediated HLX silencing. CDNA (3.5 ng) from SGHPL-4 and HTR-8/SVneo cells transfected with $H L X$-siRNA and the mock control were amplified for 40 cycles including denaturation at $95^{\circ} \mathrm{C}$ for 15 seconds and annealing/extension at $60^{\circ} \mathrm{C}$ for 60 seconds (using prevalidated Taqman gene expression assays from Applied Biosystems). Gene expression quantitation for the housekeeping gene GAPDH was coamplified with each $H L X$ target gene. Relative quantification of RB1, MYC. and EGR1 (A, B, and $\mathbf{C}$, respectively) expression in SGHPL-4 and HTR-8 cells normalized to GAPDH was calculated according to the $2^{-\Delta \Delta C T}$ method of Livak and Schmittgen, ${ }^{40}$ using the mock control as a calibrator. Significance at ${ }^{*} P<0.001(n=3$, $t$-test). The black and gray bars represent the SGHPL- 4 and HTR- $8 /$ SVneo cell lines, respectively. 
signed to target different regions of the HLX CDNA as described in Materials and Methods. HLX-si3 and HLX-si4 were chosen for this study, because they resulted in a maximal decrease of HLX expression in both SGHPL-4 and HTR-8/SVneo cell lines from our previous experiments. ${ }^{29}$ The HLX gene was inactivated at the MRNA and protein levels, in cultured trophoblast cells as described previously. ${ }^{29}$ PCR array for identification of potential HLX downstream targets was performed using the human MAPK pathway specific array. Gene expression (fold change) was calculated relative to five different housekeeping genes included in the array. The relative mRNA expression of the 84 predispensed genes following siRNA-mediated reduction of HLX expression is shown in Figure 1. Of the 84 genes in the array, genes that showed significantly altered mRNA levels with HLX inactivation using two individual HLX siRNAs were prioritized as candidate downstream targets of HLX. As shown in Figure 2, retinoblastoma-1 (RB1), myelocytomatosis viral oncogene homolog $(M Y C)$, and early growth response-1 (EGR-1) mRNA levels were highly increased with positive fold changes of $35.41,20.57$, and 13.98 , respectively. On the other hand, cyclin-dependent kinase inhibitor-1C (CDKN1C), ELK1 (a member of the ETS oncogene family), cyclin B1 (CCNB1), and JUN oncogene mRNA levels were substantially decreased as a result of $H L X$ gene reduction, with negative fold changes of $38.7,25.29$, 24.98 , and 15.55 respectively.

\section{Validation of Prioritized HLX Target Genes in Independent Trophoblast Cell Lines}

The prioritised $H L X$ downstream target genes identified from PCR-array were further validated by individual realtime PCR using independent gene-specific PCR primers (validated Taqman Assays), in both trophoblast cell lines SGHPL-4 and HTR-8/SVneo, using the two siRNA oligonucleotides HLX-si3 and HLX-si4. The independent validation results were consistent with the PCR-array. RB1, $M Y C$, and EGR-1 mRNA levels were significantly increased ( $n=3, P<0.001, t$-test) (Figure 3$)$, whereas CDKN1C, ELK1, CCNB1, and JUN mRNA levels were significantly decreased $(n=3, P<0.001, t$-test) with $H L X$ inactivation using $H L X$-si3 and $H L X$-si4, in both the cell lines tested (Figure 4).

\section{mRNA Expression of HLX Target Genes in Human Idiopathic FGR}

Expression of the HLX downstream target genes identified in cultured trophoblast cells were tested in FGR-
A

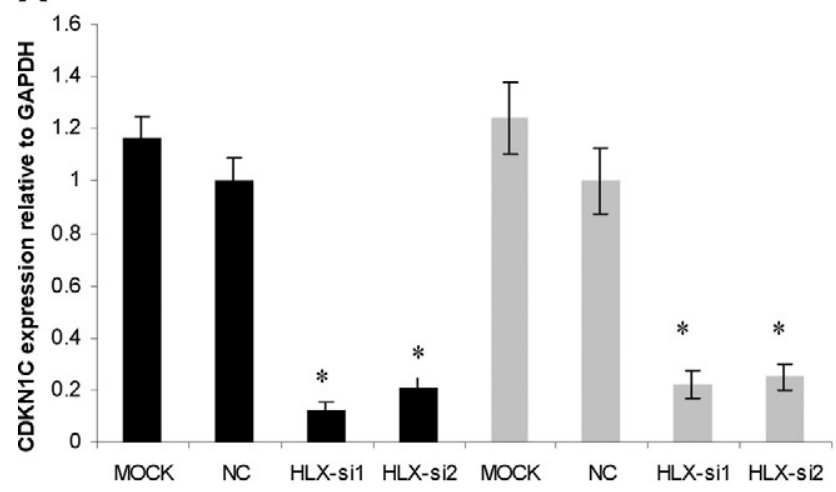

C

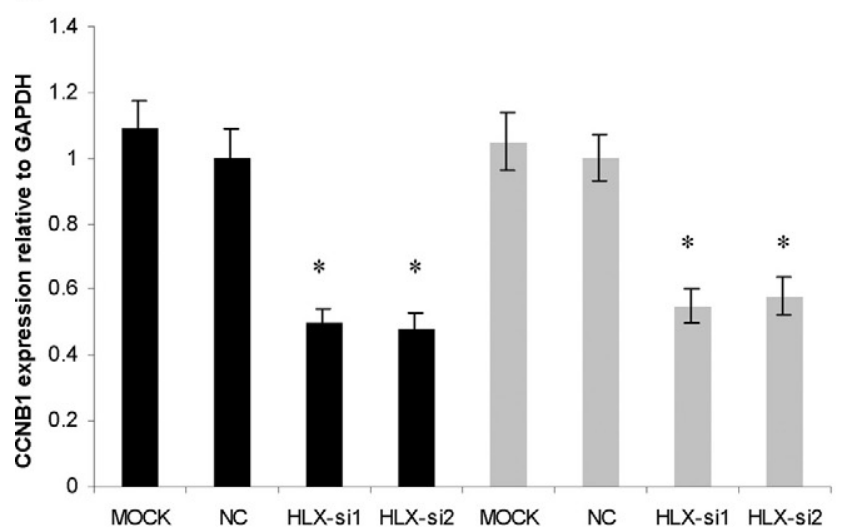

B

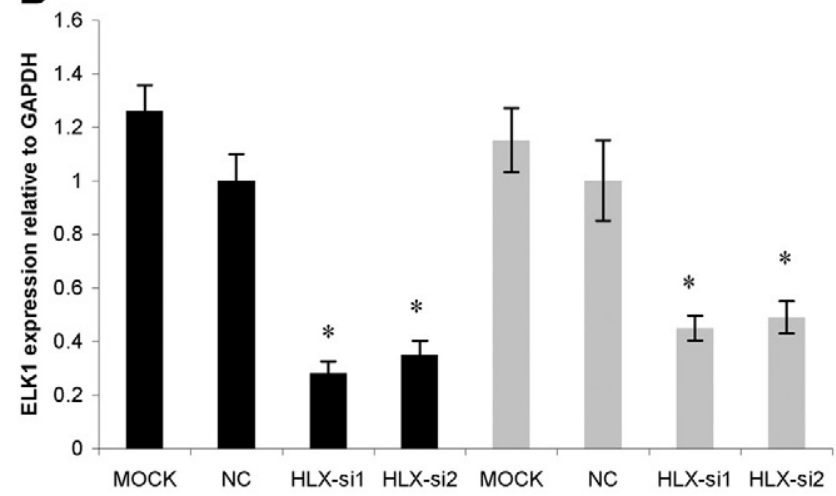

D

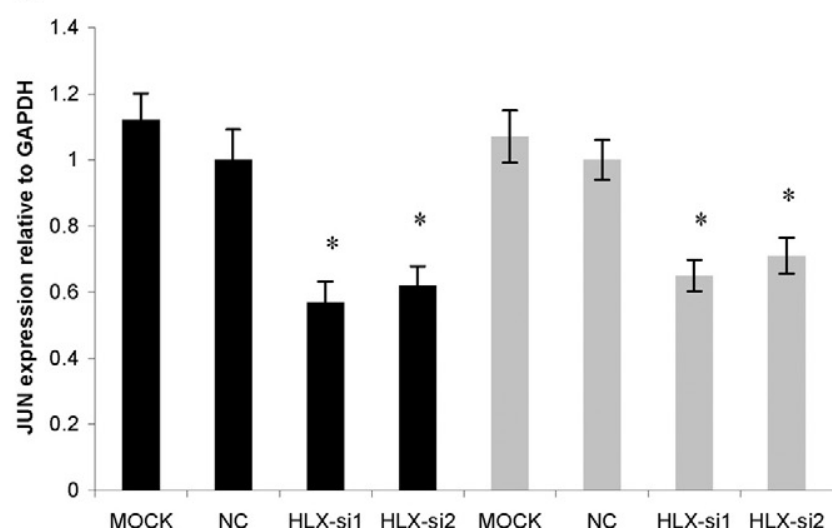

Figure 4. Validation of target genes that showed decreased expression following siRNA-mediated HLX silencing. Relative quantification of CDKN1C, ELK1, CCNB1, and JUN (A-D) expression in SGHPL-4 and HTR-8/SVneo cells normalized to GAPDH was calculated according to the $2^{-\Delta \Delta C T}$ method of Livak and Schmittgen, ${ }^{40}$ using the mock control as a calibrator. Significance at ${ }^{*} P<0.001(n=3, t$-test). The black and gray bars represent the SGHPL- 4 and HTR-8/SVneo cell lines, respectively. 

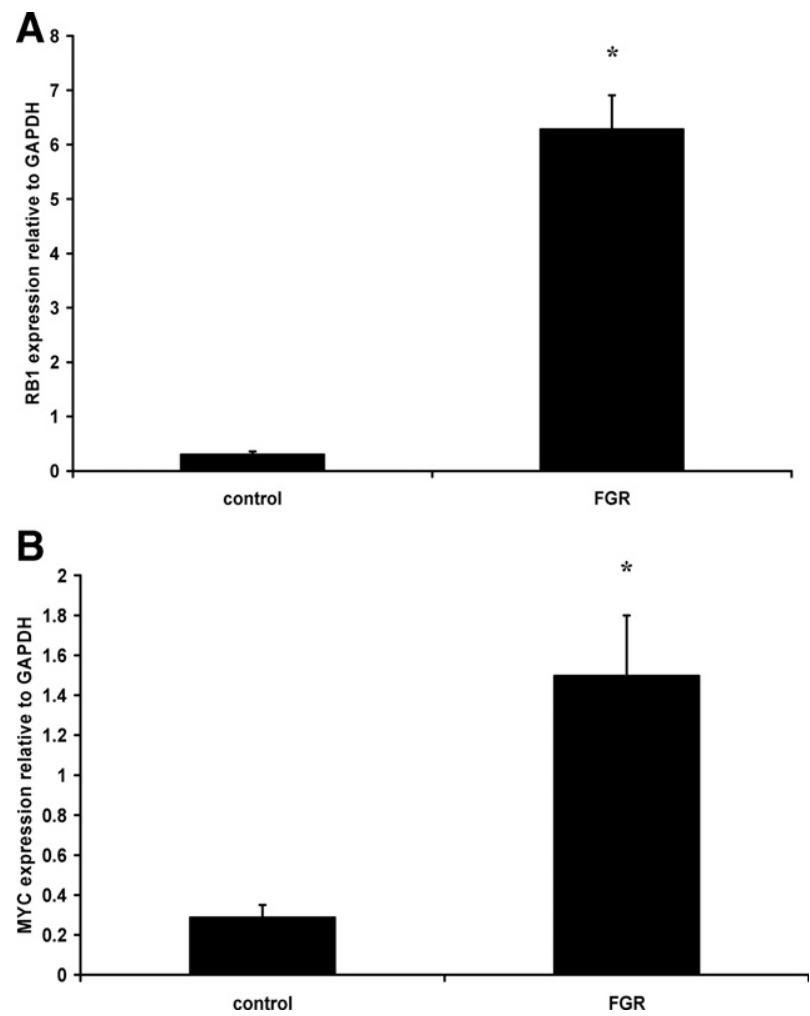

Figure 5. Increased $H L X$ target gene expression in FGR-affected placentae. Real-time PCR for relative quantitation of $R B 1(\mathbf{A})$ and $M Y C(\mathbf{B})$ normalized to the housekeeping gene GAPDH were performed in all gestation-matched controls $(n=25)$ and FGR-affected placentae $(n=25)$. Data were analyzed according to the $2^{-\Delta \Delta C T}$ method of Livak and Schmittgen. ${ }^{40}$ Statistical comparisons were performed using $t$-test. Significance at ${ }^{*} P<0.001$.

affected placentae compared with gestation agematched control placentae. As shown in Figure 5, RB1 and MYC mRNA levels were significantly increased $(n=$ 25, $P<0.001$, t-test) whereas CCNB1, ELK1, JUN, and CDKN1C mRNA levels were significantly decreased $(n=$
25, $P<0.001$, $t$-test) (Figure 6). However, the mRNA level of EGR-1 was unchanged in FGR-affected placentae compared with controls ( $4.8 \pm 0.77$ control versus $4.55 \pm$ $0.93 \mathrm{FGR}, n=25, P=0.83$, $t$-test).

\section{Protein Expression of RB1 and CDKN1C in Human Idiopathic FGR}

The expression of RB1 and CDKN1C, which showed the highest increase and decrease in mRNA levels, respectively, in FGR-affected placental tissues, was further assessed at the protein level. As shown in Figure 7, RB1 protein expression was significantly increased in FGRaffected placentae ( $n=6, P<0.001$, $t$-test) (Figure 7A), whereas CDKN1C protein expression was significantly decreased in FGR-affected placentae compared with gestation-matched controls, relative to the housekeeping gene GAPDH ( $n=6, P<0.001$, $t$-test), which was consistently expressed in all samples used (Figure 7B).

\section{Discussion}

Homeobox genes control transcription by binding to the regulatory elements in the promoter regions of their target genes. These downstream targets of homeobox genes often perform specialized roles in controlling various cell functions. Therefore, homeobox target genes are referred to as genes that act downstream of the homeobox, which may be involved, directly or indirectly, in the mediation of homeobox gene function.

In this study, a well-defined group of human pregnancies with severe idiopathic FGR, which is frequently associated with uteroplacental insufficiency, were used. These samples have been used in gene expression studies from this laboratory. ${ }^{30,34}$ The pregnancies complicated by FGR are at risk due to poor placental function ${ }^{41}$
A

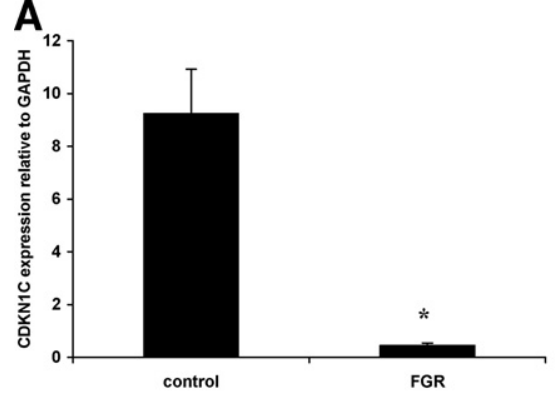

C

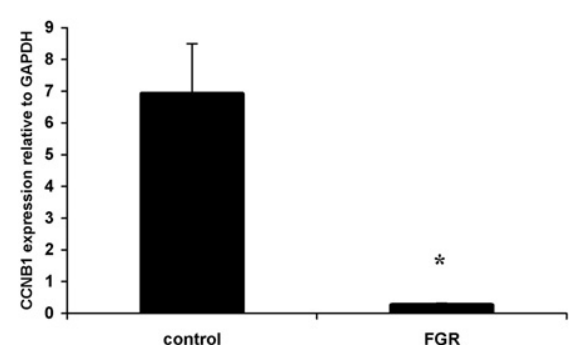

B

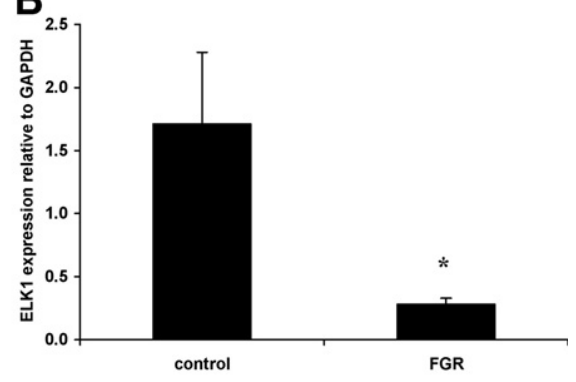

D

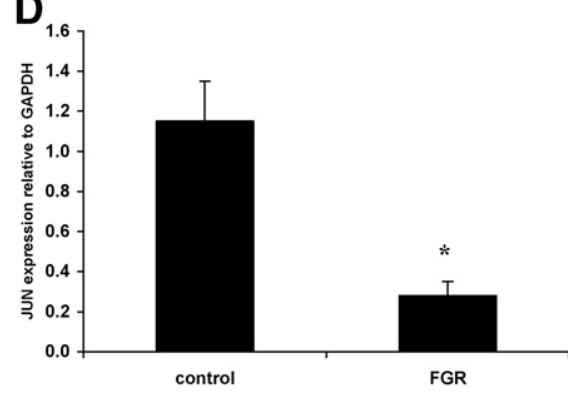

Figure 6. Decreased $H L X$ target gene expression in FGR-affected placentae. Relative quantitation of CDKN1C, ELK1, CCNB1, and JUN $(\mathbf{A}-\mathbf{D})$ normalized to the housekeeping gene GAPDH were performed in all controls $(n=25)$ and FGR-affected placentae $(n=25)$. Real-time PCR data were analyzed according to the $2^{-\Delta \Delta C 1}$ method of Livak and Schmittgen. ${ }^{40}$ Statistical comparisons were performed using $t$-test. Significance at ${ }^{*} P<0.001$. 

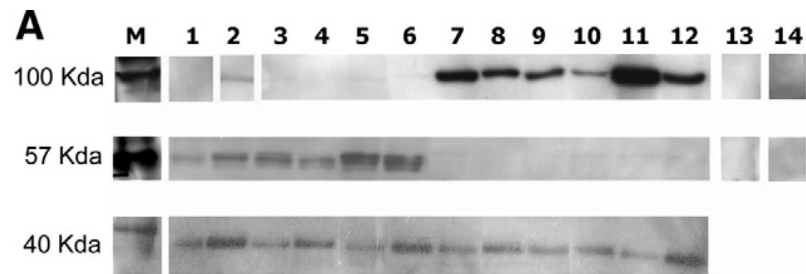

B

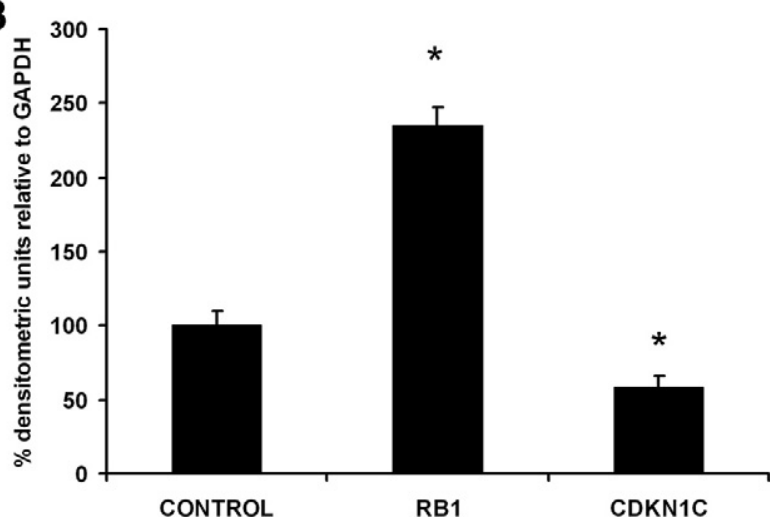

Figure 7. HLX target protein expression in FGR-affected placentae. Following protein extraction of human idiopathic FGR-affected placentae $(n=6)$ and control placentae $(n=6)$, protein assays were performed. Protein samples $(25 \mu \mathrm{g})$ were then electrophoresed and transferred to a nitrocellulose membrane. Immunoblotting was performed using commercially available $R B 1, C D K N 1 C$, and $G A P D H$ rabbit polyclonal antibodies. Composite images of autoradiographic detection of RB1 (100 kDa), CDKN1C (57 kDa), and $G A P D H(40 \mathrm{kDa})$ are shown in $\mathbf{A}$. Lanes $1-6$ are control placentae, whereas lanes 7-12 are FGR-affected placental samples. Lanes 13 and 14 are the primary antibody omitted and rabbit serum negative controls, respectively. B: The densitometric quantitation of $R B 1$ and $C D K N 1 C$ in $\mathbf{A}$, as the percentage densitometric values relative to GAPDH. Significance at ${ }^{*} P<$ $0.001, n=6, t$-test. Where indicated by white dividing lines, lanes 1 and 2 are from different areas of the same blot, whereas lanes 13 and 14 are from independent blots.

and are characterized by asymmetric growth of the fetus, altered umbilical artery diastolic velocities, and reduced liquor volume. ${ }^{6,42}$ Typically, the placentae are smaller than controls and have a variety of morphological and functional defects. Hence, in this study, the expression levels of $H L X$ target genes were determined in placental samples that meet the clinical selection criteria for FGR. ${ }^{30}$

Previous studies have shown that reduced trophoblast proliferation $^{41}$ and increased apoptosis ${ }^{43}$ occur in the villous core of FGR-affected placentae, whereas decreased trophoblast invasion ${ }^{44}$ and migration ${ }^{45}$ are seen in the FGR placental bed. We have previously shown that siRNA-mediated HLX inactivation significantly decreases trophoblast proliferation in the SGHPL-4 and HTR8-SV/ neo cell lines by up to $80 \%$, compared with mock-transfected cells, and that $H L X$ is a mediator of the cytokine colony-stimulating factor-1-dependent trophoblast proliferation. ${ }^{29}$ We have also shown that trophoblast migration significantly decreases by $70 \%$ with HLX-siRNA transfection and that $H L X$ is a mediator of hepatocyte growth factor-dependent trophoblast migration, but siRNA-mediated $H L X$ inactivation does not affect trophoblast invasion (our unpublished data). Therefore, a functional correlation between HLX and trophoblast function is evident and the placental samples used in this study reflect the functional activity of HLX in the human placenta (ie, trophoblast proliferation and migration).
A key pathway in trophoblast function is the MAPK pathway. ${ }^{33}$ This study has shown that reduced HLX expression in cultured trophoblast cells significantly alters genes in the MAPK pathway. Therefore, HLX may play an important role in directly signaling trophoblast function via the MAPK pathway, which consists of several tumor suppressor genes and cell cycle regulators.

In this study, we have identified cell cycle regulatory genes as downstream targets of the homeobox gene HLX in cultured trophoblast cells, namely RB1, MYC, EGR1, CDKN1C, ELK1, CCNB1, and JUN. RB1 and MYC mRNA expression was increased with HLX inactivation, whereas EGR1, CDKN1C, ELK1, CCNB1, and JUN mRNA expression was decreased compared with mock-transfected control cells. These findings are not only consistent in two independent trophoblast cell lines, SGHPL-4 and HTR-8/ SVneo, but also reflected in FGR-affected human placental tissue that is associated with abnormal trophoblast function. RB1 and MYC mRNA expression was significantly increased in idiopathic FGR placentae, whereas CDKN1C, ELK1, CCNB1, and JUN expression was significantly decreased. Although a significant increase in EGR-1 mRNA expression in HLX-inactivated cultured trophoblast cells was observed, EGR-1 expression was not significantly altered in idiopathic FGR placental tissue compared with control placentae. This difference in EGR-1 expression between cultured trophoblast cells and human placental tissue suggests that multiple cell types present in the whole placenta may regulate EGR-1 expression.

Various studies have shown that RB1, MYC, EGR1, CDKN1C, ELK1, CCNB1, and JUN are expressed in human extravillous trophoblast cells and are implicated in

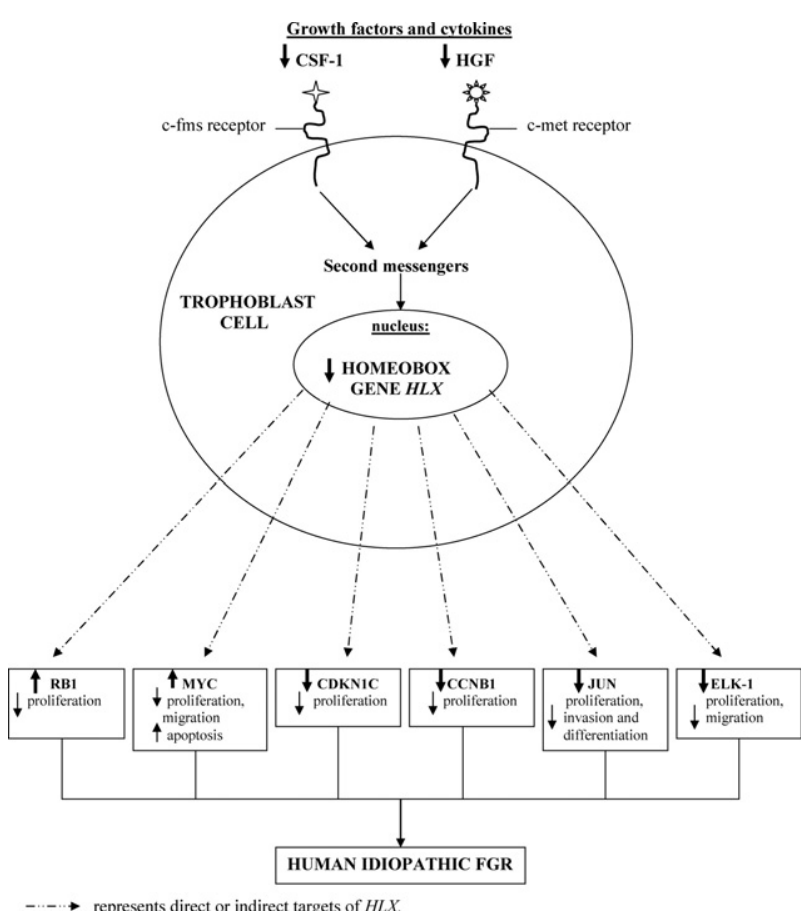

Figure 8. Schematic diagram: Summary of $H L X$ downstream target gene expression changes that alter trophoblast cell functions associated with human idiopathic FGR 
the regulation of cell proliferation and migration. ${ }^{46-52}$ This is consistent with our finding of HLX expression in the human placenta in actively proliferating and migrating trophoblast cells. ${ }^{28}$ Results from this study suggest that $R B 1, M Y C, E G R 1, C D K N 1 C, E L K 1, C C N B 1$, and JUN are direct or indirect targets of homeobox gene $H L X$ and that $H L X$-mediated target gene expression in trophoblast cells may cause the reduction in trophoblast proliferation and in migration associated with idiopathic FGR. The gene expression changes of $H L X$ downstream targets in trophoblast cells, and their interrelationship with human idiopathic FGR is summarized in Figure 8.

Mouse knockout studies for HLX target genes identified in this study provide evidence that $H L X$ target gene mutations directly result in an FGR-like phenotype due to placental defects. Targeted disruption of c-myc gene (homolog of MYC) in the mouse model system results in severe placental defects and embryonic death due to placental insufficiency. ${ }^{53}$

The product of the RB1 gene is a nuclear phosphoprotein that may act as an inhibitor of cell proliferation. ${ }^{54} \mathrm{Wu}$ et $\mathrm{al}^{55}$ have demonstrated that reduction of $R B 1$ gene expression in the mouse model system results in excessive proliferation of trophoblast cells and a severe disruption of the normal labyrinth architecture in the placenta. This is accompanied by a decrease in vascularization and a reduction in placental transport function and ultimately embryonic death. ${ }^{55}$ In our results, $R B 1$ showed the highest increase in mRNA levels in FGRaffected placentae compared with control placentae, and this was shown to be reflected in increased protein levels. Therefore, increased RB1 expression levels in FGR may reduce trophoblast proliferation and result in a fewer number of trophoblast cells available to migrate and invade into the maternal decidua. This reduction in trophoblast proliferation may lead to the shallow, inadequate remodeling of the maternal spiral arteries associated with FGR.

Studies have shown that targeted disruption of CDKN1C in the mouse model system results in severe placental defects. ${ }^{56}$ CDKN1C knockout mice have displayed an array of pre-eclampsia-like symptoms, including placental abnormalities, hypertension, proteinuria, and premature labor. ${ }^{56}$ CDKN1C is a maternally imprinted gene that is important in the regulation of embryonic implantation and development, placental growth, and the pathogenesis of proliferative trophoblastic diseases. ${ }^{48}$ This is consistent with our data, suggesting that $H L X$-mediated reduction of CDKN1C expression may reduce trophoblast proliferation. Because CDKN1C was the most decreased gene in FGR out of the prioritized $H L X$ target genes, its expression was also confirmed at the protein level. These results show that CDKN1C protein expression is also significantly decreased in idiopathic human FGR placentae compared with controls. Therefore, the reduced levels of HLX may directly or indirectly cause the reduction in CDKN1C mRNA and protein expression seen in idiopathic FGR.

Most importantly, the four HLX downstream target genes CCNB1, MYC, CDKN1C, and JUN, previously identified as $H L X$ target genes in hematopoietic progenitor cells, ${ }^{24}$ were confirmed as HLX targets in cultured trophoblast cells in this study. Therefore, this study has demonstrated that the HLX homeobox gene targets cell cycle regulatory genes in two independent cell types, and these targets are significantly altered in human idiopathic FGR placentae compared with gestation age-matched controls.

In conclusion, this is the first study to identify downstream targets of a homeobox gene in the human placenta. This study shows that candidate downstream target genes of the homeobox gene HLX are significantly altered in human idiopathic FGR-affected placentae, compared with gestation-matched control placentae from uncomplicated pregnancies. Most importantly, the findings of this study demonstrate that in vitro models for siRNA-mediated knockdown of $H L X$ expression in cultured trophoblast cells show consistent changes to those observed in human idiopathic FGR where HLX levels are reduced. These results suggest that reduced levels of $H L X$ seen in FGR cause direct or indirect effects on target genes that have been shown to be altered in FGR. Therefore, reduced HLX levels directly or indirectly cause gene expression changes in targets that have deleterious effects on trophoblast function.

\section{Acknowledgments}

We thank the Clinical Research Midwife Ms. Susan Nisbet for the collection of placental tissues and Dr. Joanne Said for the characterization of clinical samples used in this study.

\section{References}

1. Rosso IM, Cannon TD, Huttunen T, Huttunen MO, Lonnqvist J, Gasperoni TL: Obstetric risk factors for early-onset schizophrenia in a Finnish birth cohort. Am J Psychiatry 2000, 157:801-807

2. Gale CR, Martyn CN: Birth weight and later risk of depression in a national birth cohort. Br J Psychiatry 2004, 184:28-33

3. Axt R, Kordina AC, Meyberg R, Reitnauer K, Mink D, Schmidt W: Immunohistochemical evaluation of apoptosis in placentae from normal and intrauterine growth-restricted pregnancies. Clin Exp Obstet Gynecol 1999, 26:195-198

4. Regnault TR, Marconi A, Smith C, Glazier J, Novak D, Sibley C, Jansson T: Placental amino acid transport systems and fetal growth restriction-a workshop report. Placenta 2006, 26(Suppl A): S76-S80

5. Kaufmann P, Black S, Huppertz B: Endovascular trophoblast invasion: implications for the pathogenesis of intrauterine growth retardation and pre-eclampsia. Biol Reprod 2003, 69:1-7

6. Kingdom J, Huppertz B, Seaward G, Kaufmann P: Development of the placental villous tree and its consequences for fetal growth. Eur J Obstet Gynecol Reprod Biol 2000, 92:35-43

7. Chaddha V, Viero S, Huppertz B, Kingdom J: Developmental biology of the placenta and the origins of placental insufficiency. Semin Fetal Neonatal Med 2004, 9:357-369

8. Arroyo JA, Winn VD: Vasculogenesis and angiogenesis in the IUGR placenta. Semin Perinatol 2008, 32:172-177

9. Ahmed A, Perkins J: Angiogenesis and intrauterine growth restriction. Baillieres Best Pract Res Clin Obstet Gynaecol 2000, 14:981-998

10. Knoefler M, Kalionis B, Huelseweh B, Bilban M, Morrish DW: Novel genes and transcription factors in placental development-a workshop report. Placenta 2000, 21(Suppl A): S71-S73

11. Hemberger M, Cross JC: Genes governing placental development. Trends Endocrinol Metab 2001, 12:162-168

12. Rossant J, Cross JC: Placental development: lessons from mouse mutants. Nat Rev Genet 2001, 2:538-548

13. Sapin V, Blanchon L, Serre AF, Lemery D, Dastugue B, Ward SJ: Use 
of transgenic mice model for understanding the placentation: towards clinical applications in human obstetrical pathologies? Transgenic Res 2001, 10:377-398

14. Cross JC, Baczyk D, Dobric N, Hemberger M, Hughes M, Simmons DG, Yamamoto H, Kingdom JC: Genes, development and evolution of the placenta. Placenta 2003, 24:123-130

15. Quinn LM, Johnson BV, Nicholl J, Sutherland GR, Kalionis B: Isolation and identification of homeobox genes from the human placenta including a novel member of the Distal-less family, DLX4. Gene 1997 187:55-61

16. Murthi P, So M, Gude NM, Doherty VL, Brennecke SP, Kalionis B: Homeobox genes are differentially expressed in macrovascular human umbilical vein endothelial cells and microvascular placental endothelial cells. Placenta 2007, 28:219-223

17. Maas R, Bei M: The genetic control of early tooth development. Crit Rev Oral Biol Med 1997, 8:4-39

18. Kraus P, Lufkin T: Mammalian DIx homeobox gene control of craniofacial and inner ear morphogenesis. J Cell Biochem 1999, Suppl 32-33:133-140

19. Rinkenberger JL, Cross JC, Werb Z: Molecular genetics of implantation in the mouse. Dev Genet 1997, 21:6-20

20. Simmons DG, Cross JC: Determinants of trophoblast lineage and cell subtype specification in the mouse placenta. Dev Biol 2005, 284:12-24

21. Quinn LM, Latham SE, Kalionis B: The homeobox genes MSX2 and MOX2 are candidates for regulating epithelial-mesenchymal cell interactions in the human placenta. Placenta 2000, 21(Suppl A):S50-S54

22. Murthi $P$, Hiden U, Rajaraman G, Liu H, Borg AJ, Coombes FJ, Desoye G, Brennecke SP, Kalionis B: Novel homeobox genes are differentially expressed in placental microvascular endothelial cells compared with macrovascular cells. Placenta 2008, 29:624-630

23. Hentsch B, Lyons I, Li R, Hartley L, Lints TJ, Adams JM, Harvey RP: HIx homeobox gene is essential for an inductive tissue interaction that drives expansion of embryonic liver and gut. Genes Dev 1996, 10:70-79

24. Kehrl JH, Deguchi Y: Potential roles for two human homeodomain containing proteins in the proliferation and differentiation of human hematopoietic progenitors. Leuk Lymphoma 1993, 10:173-176

25. Morrish DW, Dakour J, Li H: Life and death in the placenta: new peptides and genes regulating human syncytiotrophoblast and extravillous cytotrophoblast lineage formation and renewal. Curr Protein Pept Sci 2001, 2:245-259

26. Slavotinek AM, Moshrefi A, Lopez Jiminez N, Chao R, Mendell A, Shaw GM, Pennacchio LA, Bates MD: Sequence variants in the HLX gene at chromosome 1q41-1q42 in patients with diaphragmatic hernia. Clin Genet 2009, 75:429-439

27. Suttner K, Ruoss I, Rosenstiel P, Depner M, Pinto LA, Schedel M, Adamski J, Illig T, Schreiber S, Von Mutius E, Kabesch M: HLX1 gene variants influence the development of childhood asthma. J Allergy Clin Immunol 2009; 123:82-88

28. Rajaraman G, Murthi P, Quinn L, Brennecke SP, Kalionis B: Homeodomain protein $\mathrm{HLX}$ is expressed primarily in cytotrophoblast cell types in the early human placenta. Reprod Fertil Dev 2008, 20:357-367

29. Rajaraman G, Murthi P, Leo B, Brennecke SP, Kalionis B: Homeobox gene $\mathrm{HLX} 1$ is a regulator of colony stimulating factor-1 dependent cell proliferation. Placenta 2007, 28:991-998

30. Murthi P, Doherty V, Said J, Donath S, Brennecke SP, Kalionis B: Homeobox gene HLX1 expression is decreased in idiopathic human fetal growth restriction. Am J Pathol 2006, 168:511-518

31. LaMarca HL, Dash PR, Vishnuthevan K, Harvey E, Sullivan DE, Morris CA, Whitley GS: Epidermal growth factor-stimulated extravillous cytotrophoblast motility is mediated by the activation of PI3-K, Akt and both p38 and p42/44 mitogen activiated protein kinases. Hum Reprod 2008, 23:1733-1741

32. Knerr I, Schubert SW, Wich C, Amann K, Aigner T, Vogler T, Jung R, Dotsch J, Rascher W, Hashemolhosseini S: Stimulation of GCMa and syncytin via CAMP mediated PKA signaling in human trophoblastic cells under normoxic and hypoxic conditions. FEBS Lett 2005 , 579:3991-3998

33. Fitzgerald JS, Busch S, Wengenmayer T, Foerster $\mathrm{K}$, de la Motte $\mathrm{T}$, Poehlmann TG, Markert UR: Signal transduction in trophoblast invasion. Chem Immunol Allergy 2005, 88:181-199

34. Murthi P, Fitzpatrick E, Borg AJ, Donath S, Brennecke SP, Kalionis B: GAPDH, 18S rRNA and YWHAZ are suitable endogenous reference genes for relative gene expression studies in placental tissues from human idiopathic fetal growth restriction. Placenta 2008, 29:798-801
35. Gauran RL, Wein P, Sheedy M, Walstab J, Beischer NA: Update of growth percentiles for infants born in an Australian population. Aust NZ J Obstet Gynaecol 1994, 34:39-50

36. Choy MY, Manyonda IT: The phagocytic activity of human first trimester extravillous trophoblast cells. Hum Reprod 1998, 13:2941-2949

37. Shiverick KT, King A, Frank H, Cartwright JE, Schneider H: Cell culture models of human trophoblast II: trophoblast cell lines a workshop report. Placenta 2001, 22(Suppl A):S104-S106

38. Chomzynski P, Sacchi N: Single-step method of RNA isolation by acid guanidinium thiocyanate-phenol-chloroform extraction. Anal Biochem 1987, 162:156-159

39. Puissant C, Houdebine LM: An improvement of the single-step method of RNA isolation by acid guanidinium thiocyanate-phenolchloroform extraction. Biotechniques 1990, 8:148-149

40. Livak KJ, Schmittgen TD: Analysis of relative gene expression data using real-time quantitative PCR and the $2^{-\Delta \Delta C(T)}$ method. Methods 2001, 25:402-408

41. Chen CP, Bajoria R, Aplin JD: Decreased vascularization and cell proliferation in placentas of intrauterine growth-restricted fetuses with abnormal umbilical artery flow velocity waveforms. Am J Obstet Gynecol 2002, 187:764-769

42. Jackson MR, Walsh AJ, Morrow RJ, Mullen JB, Lye SJ, Ritchie JW: Reduced placental villous tree elaboration in small-for-gestation age pregnancies: relationship with umbilical artery doppler waveforms. J Obstet Gynecol 1995, 172(2 Pt 1):518-525

43. Huppertz B, Kadyrov M, Kingdom JC: Apoptosis and its role in the trophoblast. Am J Obstet Gynecol 2006, 195:29-39

44. Kaufmann P, Black S, Huppertz B: Endovascular trophoblast invasion: implications for the pathogenesis of intrauterine growth retardation and preeclampsia. Biol Reprod 2003, (69) 1:1-7

45. Burrows TD, King A, Loke YW: Trophoblast migration during human placental implantation. Hum Reprod Update 1996, 2:307-321

46. Quenby S, Brazeau C, Drakeley A, Lewis-Jones DI, Vince G: Oncogene and tumour suppressor gene products during trophoblast differentiation in the first trimester. Mol Hum Reprod 1998, 4:477-481

47. Roncalli M, Bulfamante G, Viale G, Springall DR, Alfano R, Comi A, Maggioni M, Polak JM, Coggi G: C-Myc and tumour suppressor gene product expression in developing and term human trophoblast. Placenta 1994, 15:399-409

48. Chilosi M, Piazzola E, Lestani M, Benedetti A, Guasparri I, Granchelli G, Aldovini D, Leonardi E, Pizzolo G, Doglioni C, Menestrina F, Mariuzzi GM: Differential expression of p57kip2, a maternally imprinted cok inhibitor, in normal human placenta and gestational trophoblastic disease. Lab Invest 1998, 78:269-276

49. Kauma S, Hayes N, Weatherford S: The differential expression of hepatocyte growth factor and met in human placenta. J Clin Endocrinol Metab 1997, 82:949-954

50. Korgun ET, Celik-Oxenci C, Acar N, Cayli S, Desoye G, Demir R: Location of cell cycle regulators cyclin B1, cyclin A, PCNA, Ki67 and cell cycle inhibitors p21, p27 and p57 in human first trimester placenta and deciduas Histochem Cell Biol 2006, 125:615-624

51. Bamberger AM, Bamberger CM, Aupers S, Milde-Langosch K, Loning T, Makrigiannakis A: Expression pattern of the activating protein-1 family of transcription factors in the human placenta. Mol Hum Reprod 2004, 10:223-228

52. Takai N, Ueda T, Narahara H, Miyakawa I: Expression of c-Ets1 protein in normal human placenta. Gynecol Obstet Invest 2006, 61:15-20

53. Dubois NC, Adolphe C, Ehninger A, Wang RA, Robertson EJ, Trumpp A: Placental rescue reveals a sole requirement for c-Myc in embryonic erythroblast survival and hematopoietic stem cell function. Development 2008, 135:2455-2465

54. Jeanblanc M, Mousli M, Hopfner R, Bathami K, Martinet N, Abbady $A Q$, Siffert JC, Mathieu E, Muller CD, Bronner C: The retinoblastoma gene and its product are targeted by ICBP90: a key mechanism in the $\mathrm{G}_{1} / \mathrm{S}$ transition during the cell cycle. Oncogene 2005, 24:7337-7345

55. Wu L, de Bruin A, Saavedra HI, Starovic M, Trimboli A, Yang Y Opavska J, Wilson P, Thompson JC, Ostrowski MC, Rosol TJ, Woollett LA, Weinstein M, Cross JC, Robinson ML, Leone G: Extra-embryonic function of $\mathrm{Rb}$ is essential for embryonic development and viability. Nature 2003, 421:942-947

56. Knox KS, Baker JC: Genome-wide expression profiling of placentas in the p57Kip2 model of pre-eclampsia. Mol Hum Reprod 2007, 13:251-263 